\title{
Arbitrary wave synthesis using spectrall transformations
}

\section{Baquero, M. Ferrando, G. Escobar, A. Navarro, P. Pastor, E. Selfa}

Departamento de Comunicaciones UPV, Camino de Vera $s / n$, 46071-Valencia (Spain)

Telephone and Fax: 3463877300,3463877309

\section{Abstract}

The different transformations among plane, cylindrical and spherical spectrum allow to tackle the arbitrary field synthesis from a set of known sources. Putting all the spectrums in the same coordinate axis we can obtain a system of equations that allows us to find the optimal source weights, which synthesize the wished field. In this paper we present how to get the minimum number of sources needed to obtain a plane wave that allows to measure antennas or radar cross section in an indoor near-field range.

\section{Introduction}

Sometimes the classical near-field far-field transformation algorithms need a lot of sample points that involve too much measurement time. In the other side, if we measured the antenna in far field it would be only necessary a probe at Rayleigh distance to assure plane wave over the antenna under test. It is logical to think that in an intermediate zone we can measure the antenna pattern using a few number of sample points, and perhaps in an indoor range (figure 1).

All the antenna measurements methods synthesize a plane wave in the volume occupied by the antenna under test, sometimes physically like in a far-field range or in a compact range, and sometimes by software like in the classical near-field far-field transformation algorithms. In these algorithms the probe weights to synthesize the plane wave are implicit. In an intermediate measurement zone we need to know the optimal weights in each measured point to get a plane wave in the volume occupied by the test antenna. This goal results easy if we use the field spectral expressions and their transformations. Expressing all the spectrum of the problem (plane wave and sources spectrums) in a same coordinates axis we obtain a system of equations; its solution will give the optimal weights.

\section{Theory}

It's known that the electromagnetic general solution in a free source region can be obtained from [1]:

$$
\begin{aligned}
& \vec{E}=-\frac{1}{\epsilon} \nabla \times \psi^{\prime} \vec{r}+\frac{1}{j \omega \mu \epsilon} \nabla \times \nabla \times \psi^{a} \vec{r} \\
& \vec{H}=\frac{1}{\mu} \nabla \times \psi^{\overrightarrow{a r}}+\frac{1}{j \omega \mu \epsilon} \nabla \times \nabla \times \psi^{\prime} \vec{r}
\end{aligned}
$$

where $\psi^{a}$ and $\psi^{f}$ will be solutions to the Helmholtz scalar equation:

$$
\nabla^{2} \psi+k^{2} \psi=0
$$

We have got the $\overrightarrow{\mathbf{r}}$ vector like electric and magnetic potentials direction in order to work with $\operatorname{TM}^{\mathrm{r}}\left(\psi^{\mathrm{a}}\right)$ and $\mathrm{TE}^{\mathrm{r}}\left(\psi^{\mathrm{f}}\right)$ modes, which result more appropriate in spherical coordinates. We will choose the elemental function proposed by Hansen [2].

$\psi_{\text {inm }}=\frac{1}{\sqrt{2 \pi}} \frac{1}{\sqrt{n(n+1)}}\left(-\frac{m}{|m|}\right)^{m} h_{n}^{(i)}(k r) \bar{P}_{n}^{|m|}(\cos \theta) e^{j m \phi}$ where $h^{(i)}$ is the spherical Hankel function and

$\overline{\mathrm{P}}|\mathrm{m}|$ is the normalized associated Legendre function. Combining these elemental functions we will be able to obtain the potentials spectral expression $\psi^{\mathrm{a}}$ y $\psi^{\mathrm{f}}$ as:

$$
\begin{gathered}
\psi^{a}=\sum_{l=1}^{\infty} e_{l}^{a} \psi_{l}=\bar{\psi} \bar{e}^{-a^{t}} \\
\psi^{f}=\sum_{l=1}^{\infty} e_{i}^{f} \psi_{l}=\bar{\psi} \bar{e}^{-\prime \prime} \\
(l-2\{(n(n+1)+m-1\}+i ; i=3,2 ; n-1, \ldots \infty ; m=-n \ldots, n)
\end{gathered}
$$

where we will name the spherical wave spectrum as $\mathrm{e}_{1}^{\mathrm{a}}$ and $\mathrm{e}_{1}^{\mathrm{f}}$. In order to use more compact expression we define the vectors $\overline{\mathrm{e}}$ and $\bar{\psi}$ like:

$$
\begin{aligned}
& \overline{e^{a}}=\left[e_{1}^{a} e_{2}^{a} \ldots e_{1}^{a} \ldots\right] \\
& \overline{e^{f}}=\left[e_{1}^{f} e_{2}^{f} \ldots e_{1}^{f} \ldots\right] \\
& \bar{\psi}=\left[\psi_{1} \psi_{2} \ldots \psi_{1} \ldots\right]
\end{aligned}
$$

We could obtain similar expressions starting from plane or cylindrical elemental waves. The knowledge of the different transformations among spectrums sketched in figure 2 will result a powerful tool to analyze electromagnetic problems.

In this paper we will use the spherical-spherical transformation applied to the antenna measurement.

Any coordinate change may be always accomplished by a sequence of rotations and translations (figure 3).

The rotations will not mean crossing between $\mathrm{TM}^{\mathrm{r}}$ and $\mathrm{TE}^{\mathrm{r}}$ modes, whereas the translation will (sketched in the figure 4): Describing the rotation with the three Euler angles $\left(\psi_{0}, \theta_{0}, \chi_{0}\right)$ will imply a spectral change given by:

$$
\begin{aligned}
& e^{{ }_{i n \mu}}=\sum_{m=-n}^{n} e_{i n m} e^{j m \phi_{0}} d_{\mu m}^{n}\left(\theta_{o}\right) e^{j \mu x_{0}} \\
& (\mu=-n, \ldots, 0, \ldots, n)
\end{aligned}
$$

where the function $\mathrm{d}_{\mu \mathrm{m}}^{\mathrm{n}}\left(\theta_{0}\right)$ can be found from Edmonds [3].

On the other hand a translation of $z_{0}$ over the $z$ axis will imply a spectral change (in the case of $\mathrm{TE}^{\mathrm{r}}$ modes) given by:

$$
\begin{aligned}
& e_{i v m}^{f}=\sum_{i=1}^{2} \sum_{\substack{n=1 m i \\
n \neq 0}}^{\infty} \frac{1}{2} e_{i n m}^{f} C_{1 m v}^{\ln (1 \cdot 2)}\left(k z_{0}\right) \\
& e_{j v m}^{a}=\sum_{i=1}^{2} \sum_{\substack{n=1 m \mid \\
n \neq 0}}^{\infty} \frac{1}{2 j} \eta e_{i n m}^{f} C_{2 m v}^{i n(i+2)}\left(k z_{0}\right) \\
& j=1,2 ; v=|m|, \ldots, \infty(v \neq 0)
\end{aligned}
$$

where the function $\mathrm{C}_{\sigma \mu \nu}^{\mathrm{sn}(\mathrm{c})}(\mathrm{kzo})$ can be find from Hansen [2]. A similar expression could be obtained starting from $\mathrm{TM}^{\mathrm{r}}$ modes.

Combining rotations and translation we can express sphericalspherical transformations $\left(\mathrm{T}_{\mathrm{ss}}\right)$

$$
\left[\bar{e}^{a}, \bar{e}^{f}\right]=T_{s s}\left[\bar{e}^{-a}, \bar{e}^{f \prime}\right]
$$




\section{System of Equations}

Expressing all the spectrums in the same coordinate system we can find a system of equations which resolves specific problems. For instance let us suppose that we need to obtain a plane wave with an arbitrary distribution source just as sketched in figure 5.

It's possible to translate every spectrum source at the main coordinate system $(\mathrm{x}, \mathrm{y}, \mathrm{z}),\left(\right.$ source $\left.\mathrm{i}^{\text {th }}: \psi_{s i}^{a}=\bar{\psi} \bar{e}_{s i}^{a}, \quad \psi_{s i}^{\prime}=\bar{\psi} \bar{e}_{s i}^{\prime}\right)$, and adding every source spectrum weighted with " $w_{i}$ " we obtain

$\psi_{T}^{a}=\sum_{i=1}^{N} w_{i}\left(\bar{\psi}_{\bar{e}}^{a_{s i}^{t}}\right)=\bar{\psi} \bar{E}^{a} \bar{w}^{t} \psi_{T}^{f}=\sum_{i=1}^{N} w_{i}\left(\bar{\psi} \bar{e}_{s i}^{\prime t}\right)=\bar{\psi} \bar{E} t \bar{w}$

where $\bar{w}=\left[w_{1} \ldots w_{N}\right]$ and $\bar{E}^{a, f}=\left[\bar{e}_{s i}^{a_{i} f} \ldots \bar{e}_{s N}^{a_{1} f}\right]$.

Making them equal to the known plane wave potentials

$$
\left(\bar{e}_{P W}^{a}, \bar{e}_{P W}^{f}\right)
$$

$$
\left\{\begin{array}{l}
\bar{e}_{P W}^{a t}=\bar{E}^{a} \bar{w}^{t} \\
\bar{e}_{P W}^{\prime \prime}=\bar{E}^{f} \bar{w}^{t}
\end{array}\right\} \Rightarrow \bar{e}^{-1}=\overline{\bar{E}} \bar{w}^{t}
$$

Note that we need to truncate the vector $\bar{\psi}$ in $M$ modes depending on the plane wave size [2].

To obtain the appropriate weights we can use de Singular-Value Decomposition method. There exist the possibility that this solution leads to a low directivity in the test zone, and this means a serious problem. To avoid that, it is necessary to introduce a constraint on the power radiated by the sources.

\section{Antenna Measurement in Fresnel Zone}

A possible application is the antenna measurement in Fresnel zone with a minimum number of sample points. For instance, if we want to measure an antenna with a diameter of $8 \lambda$, the
Rayleigh distance will be $128 \lambda$, and perhaps longer than the anechoic chamber maximum dimension, having to measure it in an outdoor range with all the associated problems. Measuring the near field in a cylindrical configuration (figure 6 ) we will need approximately $\mathrm{N}_{\mathrm{z}} \times \mathrm{N}_{\phi}=32 \times 50$ measured points.

In a cylindrical system with continuous movement in $\phi$, the number of points in $\phi$ does not imply more measurement time, but the number in $\mathrm{z}$ can be critical. If we can measure in an indoor range at $70 \lambda$ only 5 points in $z$ are necessary.

The optimal weights for a $5 \times 5$ cylindrical array with elemental dipoles with $\Delta z=4 \lambda$ and $\Delta \phi=2^{\circ}$, after solving the system of equations will have the aspect shown in figures $7 \mathrm{a}$ and $7 \mathrm{~b}$.

The plane wave synthesized with these weights is shown in figures $8 \mathrm{a}$ and $8 \mathrm{~b}$.

If we measure a hypothetical antenna of $D=8 \lambda$ over a cylindrical surface we can get its pattern making the convolution between the calculated weights and the measured field. The results appear in the figures 9 and 10 .

\section{Conclusion}

It is possible to use the spectrum translation to obtain a system of equations, that allows us to synthesize a plane wave, or any wave in near field, getting antenna measurements in an indoor range with the minimum number of points. For instance it is possible to measure the antenna pattern, with $\mathrm{D}=20 \lambda$, at $\frac{\mathrm{D} 2}{\lambda}$ with 3 sources or at $1 / 2 \frac{D 2}{\lambda}$ with 7 sources. In near field 40 sources would be needed.

1. Harrington, R.F.; Time-Harmonic Electromagnetic F'ields; 1961; McGraw-Hill Book Company, Inc. New York

2. Hansen, J.E.; Spherical near-field antenna measurements; 1988; Peter Peregrinus Ltd. IEE

3. Edmonds, A.R.; Angular momentum in quantum mechanics; 1974; Princeton University Press, Princeton, New Jersey
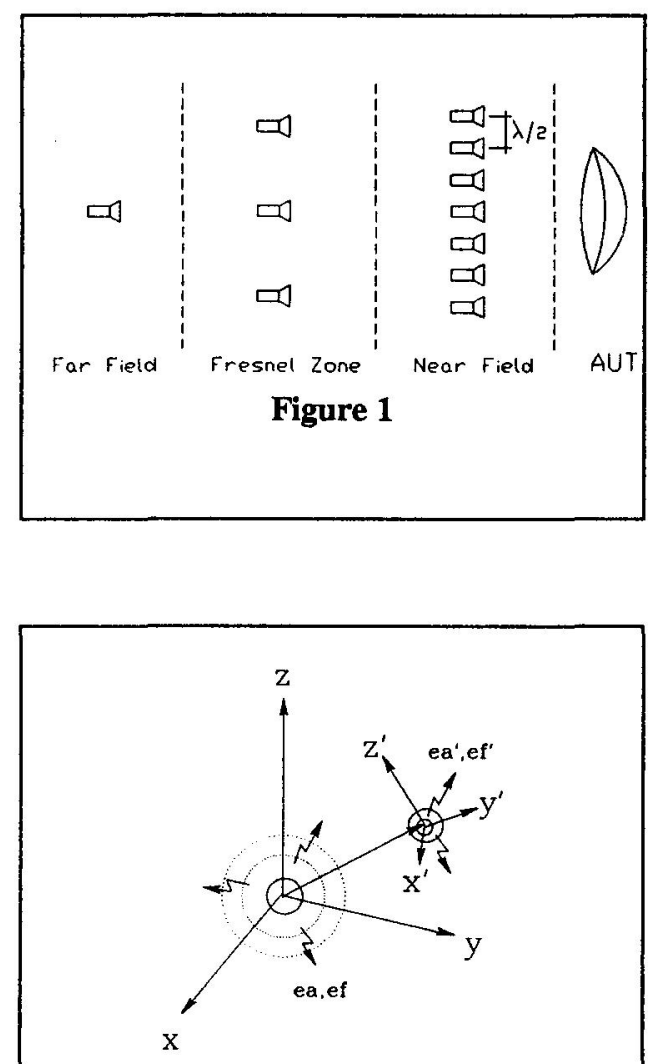

Figure 3
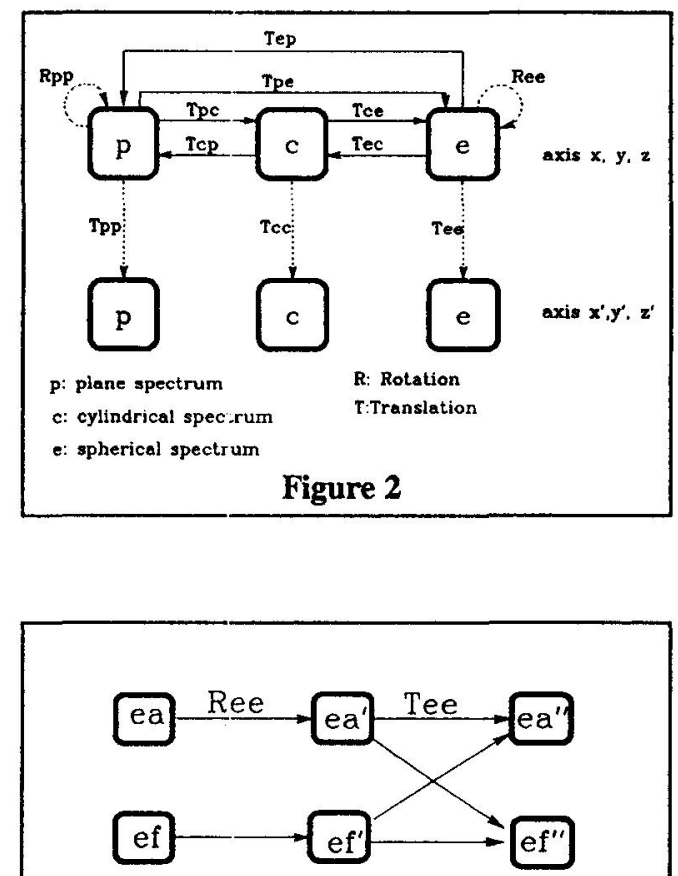

R: Rotation

T: Translation

Figure 4 